\title{
Evaluation of mode vector for near-field 2-D target imaging using Khatri-Rao product extended array processing
}

\author{
Honoka Hazawa $^{1 \text { a) }}$, Hiroyoshi Yamada ${ }^{1}$, and Hiroki Mori ${ }^{2}$ \\ ${ }^{1}$ Graduate School of Science \& Technology, Niigata University, \\ Ikarashi 2-8050, Nishi-Ku, Niigata 950-2181, Japan \\ ${ }^{2}$ Toshiba Corporation, \\ 1, Komukaitoshiba-cho, Saiwai-ku, Kawasaki 212-8585, Japan \\ a) hazawa@wave.ie.niigata-u.ac.jp
}

\begin{abstract}
Achieving high-precision and high-resolution imaging with millimeter-wave radar requires a large number of antennas and data volumes. In this study, we applied the Khatri-Rao (KR) product extended array processing to synthetic aperture radar (SAR) data to increase the number of antenna elements substantially and extend the synthetic aperture length. The target of interest in this study is relative short range target, which is several meters away from the radar, such as indoor human with a small object on his/her body. When the target is close to the radar, target imaging by SAR using the far-field approximation will often increase the error. In this case target-imaging by using range-migration is required. However, this processing often computationally inefficient. In this study, we evaluate image-quality of short-range 2-dimensional imaging with far-field and near-field mode vector with/without range migration. The image-quality is examined by peak power gain. Numerical results show that the near-filed mode vector works properly for out study without range migration. In addition, SAR imaging using KR product extended arrays shows that image quality can be improved compared to conventional uniform SAR.
\end{abstract}

Keywords: short-range target imaging, Khatri-Rao product, SAR, millimeter-wave radar, low-redundancy linear arrays

Classification: Antennas and Propagation

\section{References}

[1] H.-M. Chen, S. Lee, R. M. Rao, M.-A. Slamani, and P. K. Varshney, "Imaging for concealed weapon detection," IEEE Signal Process. Mag., vol. 22, no. 2, pp. 52-61, Mar. 2005. DOI:10.1109/MSP.2005.1406480

[2] J. M. Lopez-Sanchez and J. Fortuny-Guasch, "3-D radar imaging using range migration technique," IEEE Trans. Antennas Propag., vol. 48, no. 5, pp. 728-737, May 2000. DOI:10.1109/8.855491

[3] Y. Wakamatsu, H. Yamada, and Y. Yamaguchi, "MIMO Doppler radar using Khatri-Rao product virtual array for indoor human detection," IEICE Trans. Commun., vol. E99-B, no. 1, pp. 124-133, Jan. 2016. DOI:10.1587/transcom. 
2015ISP0029

[4] M. Soumekh, Synthetic Aperture Radar Signal Processing with MATLAB Algorithms, Wiley Interscience, 1999.

[5] A. Camps, A. Cardama, and D. Infantes, "Synthesis of large low-redundancy linear arrays," IEEE Trans. Antennas Propag., vol. 49, no. 12, pp. 1881-1883, Dec. 2001. DOI:10.1109/8.982474

\section{Introduction}

In recent years, applications of millimeter-wave (MW) radar for indoor human monitoring and security are expected [1]. In particular, fears of terrorism and suspicious person or objects are increasing, and the importance of a system to check the possession of dangerous materials is being reviewed. In this study, by applying Khatri-Rao (KR) product extended array processing for Synthetic Aperture Radar (SAR) data, we increased the number of antenna elements virtually and extended the synthetic aperture length. By using KR processing, the imaging with a small number of antenna elements became possible.

In addition, when acquiring 3D or 2D imaging for short-distance targets, it is necessary to consider near-field effect, since far-filed approximation no longer holds [2]. If signal processing is performed using far-field approximation in such a situation, the imaging quality deteriorates and spurious responses often occur. Of course, joint estimation [3] based on strict location for 2D/3D imaging can yield good results, but it is computationally inefficient. Since we can use enough frequency bandwidth in the MW-FMCW radar, it is preferable to make $2 \mathrm{D} / 3 \mathrm{D}$ target image after delay estimation.

In this report, we evaluated image quality of $2 \mathrm{D}$ estimation for short-range target by using SAR. In the imaging algorithm employed here, we first applied FFT to beat signal in each measurement point for distance estimation, then we carried out direction estimation. The image quality discussed here is concerned with a suitable mode vector in the direction (or azimuth location) estimation. We show imaging results of point targets by using far-field mode vector, near-field mode vector, and strict mode vector with range migration [4]. To evaluate the results quantitatively, peak power loss of target response was employed. From these results, we show that the imaging accuracy of the near-field mode vector without range migration could be acceptable for relative short-range target of our interests. In addition, SAR imaging using KR product virtual arrays could improve image quality in compared to that by the conventional uniform SAR.

\section{Receiving data model}

In this study, we considered 2D imaging, which is azimuth and range direction (or $x-y$ plane), by one-dimensional scanning SAR. We also assumed that the range/ distance estimation in each measurement point was done by FFT for simplicity. In the followings, we only focused on the direction/azimuth position estimation by using the array data by the range of interest. 
Let us assume that a linear array of $L$ data points obtained from $i$-th range bin having one target response for simplicity. The received signal $\boldsymbol{x}_{i} \in \mathbb{C}^{L}$ of the plane wave of complex amplitude $s$ coming from the $\theta$ direction is given by

$$
\boldsymbol{x}_{i}=\boldsymbol{a}(\theta) s+\boldsymbol{n},
$$

where $\boldsymbol{n}$ is the additive noise, and $\boldsymbol{a}(\theta) \in \mathbb{C}^{L}$ denotes the response of the array to the plane wave of arriving from direction $\theta$. If the target is located in the far-field area, we can denote $\boldsymbol{a}(\theta)$ by using Far-field mode vector as follows:

$$
\boldsymbol{a}(\theta)=\left[1, e^{-j \frac{2 \pi}{\lambda} 2 \Delta \mathrm{d} \sin \theta}, e^{-j \frac{2 \pi}{\lambda} 4 \Delta \mathrm{d} \sin \theta}, \cdots, e^{-j \frac{2 \pi}{\lambda} 2(\mathrm{~L}-1) \Delta \mathrm{d} \sin \theta}\right]^{T},
$$

where $\Delta \mathrm{d}$ is array spacing. Note that the phase constant becomes doubled as compared to the mode vector in conventional array signal processing since SAR data acquisition is assumed in this study. When there exist $K$ targets, it becomes:

$$
\begin{gathered}
\boldsymbol{x}_{i}=\sum_{k=1}^{K} \boldsymbol{a}\left(\theta_{k}\right) s_{k}+\boldsymbol{n}=\boldsymbol{A} \boldsymbol{s}+\boldsymbol{n}, \\
\boldsymbol{A}=\left[\boldsymbol{a}\left(\theta_{1}\right), \boldsymbol{a}\left(\theta_{2}\right), \cdots, \boldsymbol{a}\left(\theta_{K}\right)\right], \\
\boldsymbol{s}=\left[s_{1}, s_{2}, \cdots, s_{K}\right]^{T},
\end{gathered}
$$

where $s_{k}(t)$ and $\theta_{k}$ are the complex amplitude and arrival direction of each arrival wave, respectively, For near-field target, we should consider the spherical wave effect in the azimuth direction estimation. There are two ways: the first one is an approximate approach to just compensate phase shift by the spherical effect. This is proportional to delay time of the target. We will also refer to $\boldsymbol{a}(\tau) \in \mathbb{C}^{L}$ as Near-field approximated mode vector, and is given by

$$
\boldsymbol{a}(\tau)=\left[e^{-j 2 \pi f_{c} \tau_{1}}, e^{-j 2 \pi f_{c} \tau_{2}}, \cdots, e^{-j 2 \pi f_{c} \tau_{L}}\right]^{T}
$$

Where $f_{c}$ is the center frequency and $\tau_{i}(i=1,2, \cdots L)$ is round trip delay time to target at each antenna. Note that the data in $\boldsymbol{x}_{i}$ is extracted in the same range-bin. This is an approximated mode vector in this sense. Strictly speaking, range migration is required for near-field target. In this case, we extract the range-bin data corresponding to the delay time (or distance) from the radar to the target. In the following analysis, we denote this estimation as "near-field mode vector with range migration."

\section{Khatri-Rao product virtual array}

Consider the case where a narrow band signal is received by the $L$ elements LowRedundancy Linear Arrays (LRLA) [5]. The received data are expressed by (3) by using corresponding array configuration, then the correlation matrix $\boldsymbol{R}_{x x}$ can be given by

$$
\begin{aligned}
\boldsymbol{R}_{x x} & =E\left[\boldsymbol{x} \boldsymbol{x}^{H}\right] \\
& =\boldsymbol{A} \boldsymbol{S} \boldsymbol{A}^{H}+\boldsymbol{R}_{N} \\
& =\sum_{k=1}^{K}\left|s_{k}\right|^{2} \boldsymbol{a}\left(\theta_{k}\right) \boldsymbol{a}\left(\theta_{k}\right)^{T}+\boldsymbol{R}_{N},
\end{aligned}
$$

where $\boldsymbol{A}$ is the mode matrix, $\boldsymbol{S}$ is the source correlation matrix, and $\boldsymbol{R}_{N}$ is the noise correlation matrix. By using a vectorization operation to the correlation matrix, we 
can obtain the following virtual array data vector. Then $v e c[\cdot]$ is a function to vectorize the matrix.

$$
\begin{aligned}
\boldsymbol{z} & =\operatorname{vec}\left[\boldsymbol{R}_{x x}\right] \\
& =\sum_{k=1}^{K}\left|s_{k}\right|^{2} \operatorname{vec}\left[\boldsymbol{a}\left(\theta_{k}\right) \boldsymbol{a}\left(\theta_{k}\right)^{H}\right]+\operatorname{vec}\left[\boldsymbol{R}_{N}\right] .
\end{aligned}
$$

This is the basic concept of KR transform. We often take out the non-overlapping elements in vec $\left[\boldsymbol{a}\left(\theta_{k}\right) \boldsymbol{a}\left(\theta_{k}\right)^{H}\right]$, and make corresponding mode vector $\boldsymbol{b}\left(\theta_{k}\right)$ as follows:

$$
\boldsymbol{z}^{\prime}=\sum_{k=1}^{K} \boldsymbol{b}\left(\theta_{k}\right)\left|s_{k}\right|^{2}+\operatorname{vec}\left[\boldsymbol{R}_{N}\right]
$$

In this way, we can obtain virtually extended data vector.

The KR processing is straightforwardly applicable for 2D joint estimation [4]. However, when we apply combination of 1D estimation as the scenario in this letter, we must estimate the range first because phase information of targets is dropped by the KR processing.

\section{Simulation results}

\subsection{Imaging quality comparison}

In this section, we show some simulation results of $2 \mathrm{D}$ position estimation using SAR data obtained by virtually expanding the synthetic aperture length by applying the KR processing. The employed array in this simulation is LRLA whose element

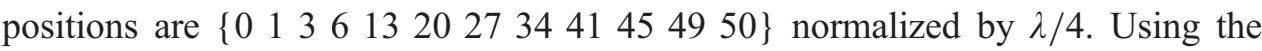
mode vector in (2) and (6), respectively, we performed 2-D target location estimation on the $\mathrm{x}-\mathrm{y}$ coordinate plane, where $x$ and $y$ corresponds to range and azimuth direction, respectively. Center Frequency of the radar is $79 \mathrm{GHz}$ and Bandwidth is $3 \mathrm{GHz}$. In these simulations, we deployed three point targets on $(X, Y)=\# 1(0.5 \mathrm{~m}, 2.5 \mathrm{~m}), \# 2(0 \mathrm{~m}, 3 \mathrm{~m})$, and \#3 (1 m, $3.5 \mathrm{~m})$.

Imaging results are shown in Figs. 1(a), (b) and (c). Figs. 1(d), (e) and (f) show the results of DOA estimation on target (\#1). We confirmed that the grating lobe could be suppressed simply by increasing the accuracy of the mode vector. Of course, the results using Near-field mode vector with range migration is the best. However, by using the Near-field mode vector without range migration, we can obtain a good imaging result in this simulation.

\subsection{Evaluation of mode vector}

To evaluate image quality quantitatively, we adopt peak power loss of point target. We got an indication of whether to use long-range mode vectors or short-range mode vectors at the distance between the target and the antenna. In this paper, we examine peak power loss in comparison to the peak power in a far-field target. Here we adopt the target at range of $40 \mathrm{~m}$ as a reference target. Propagation loss due to range distance is compensated in the following evaluation. We compared the antenna aperture lengths and compared the case of the Uniform Linear Array (ULA) without KR processing and the case of LRLA with KR processing. The 


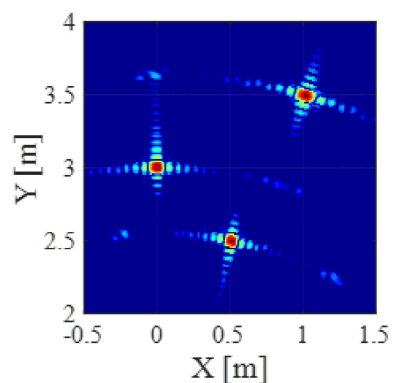

(a) Position estimation using Far-field mode vector $\mathrm{w} / \mathrm{KR}$, w/o range migration.

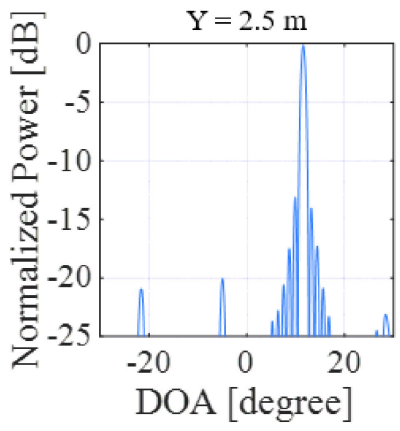

(d) DOA estimation using Far-field mode vector $\mathrm{w} / \mathrm{KR}$, w/o range migration

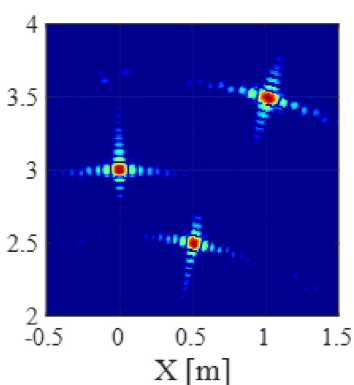

(b) Position estimation using Near-field mode vector $\mathrm{w} / \mathrm{KR}$, w/o range migration.

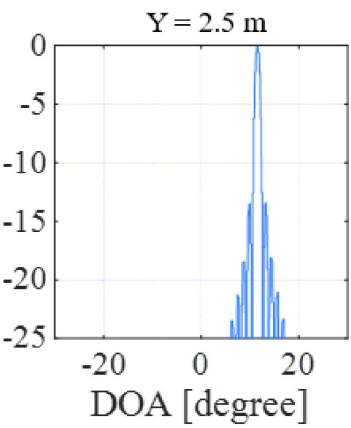

(e) DOA estimation

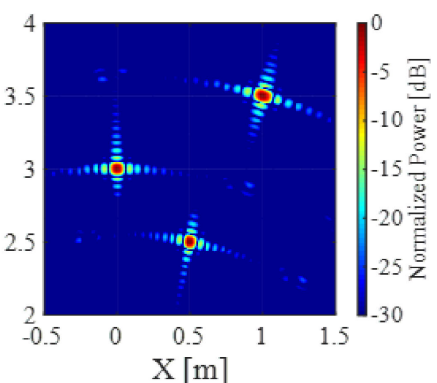

(c) Position estimation using Near-field mode vector w/ KR, w/ range migration.

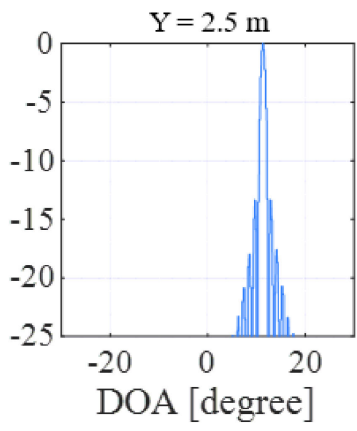

(f) DOA estimation using Near-field mode vector using Near-field mode vector $\mathrm{w} / \mathrm{KR}, \mathrm{w} / \mathrm{o}$ range migration. w/ KR, w/ range migration.

Fig. 1. Comparison of imaging accuracy.

FM-CW radar parameter used here is Center Frequency of $79 \mathrm{GHz}$, Sweep Time of 5 msec., and frequency bandwidth of $1 \mathrm{GHz}$ and $3 \mathrm{GHz}$, respectively. In this simulation, we assume a point target at $(x, \mathrm{y})=(0 \mathrm{~m}, 0 \sim 10 \mathrm{~m})$ at $1 \mathrm{~m}$ intervals in $y$. In Fig. 2, we show the simulation parameters. The LRLA element arrangements are selected to realize the same virtual aperture length to the corresponding ULA. The simulation results of evaluation mode vector is shown in Fig. 3, where (a) and (c) show the results of the Far-filed mode vector, and (b) and (d) show the results of the Near-field mode vector. Figs. 3(a) and (b) show the results with ULA. On the other hand, Figs. 3(c) and (d) show the results of the LRLA with the KR precessing.

From these results, we have confirmed that even if the aperture length is large, the use of the near-field mode vector can suppress the deterioration of accuracy. It was also found that the accuracy is better for the virtual arrays by the KR processing having the same aperture length equivalently. In addition, we should

(a) Antenna elements arrangement.

\begin{tabular}{cc}
\hline & $\begin{array}{c}\text { Element spacing: } \lambda / 4 \mathrm{~mm} \\
\text { ULA : approx. } 9,19,28 \mathrm{~cm} \\
\text { LRLA w/o KR: approx. } 5,10,14 \mathrm{~cm} \\
\text { LRLA w/ KR: approx. } 9,19,28 \mathrm{~cm}\end{array}$ \\
& \\
ULA $:$ & $0 \sim 100$ \\
& $0 \sim 200$ \\
& $0 \sim 300$ \\
LRLA $:$ & 01361320273441454950 (12 elements) \\
& 01281420314253647586919699100101 (17 elements) \\
0134561116213549637791105119128132141144145 (21 elements) \\
\hline
\end{tabular}

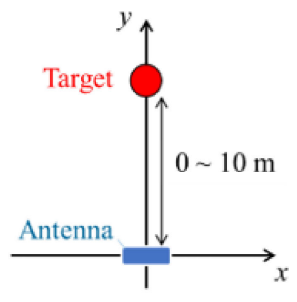

(b) Target position.

Fig. 2. Simulation parameters. 
note that the accuracy depends on the frequency bandwidth of the MW-radar. Image deterioration becomes severe for wide-bandwidth radar.

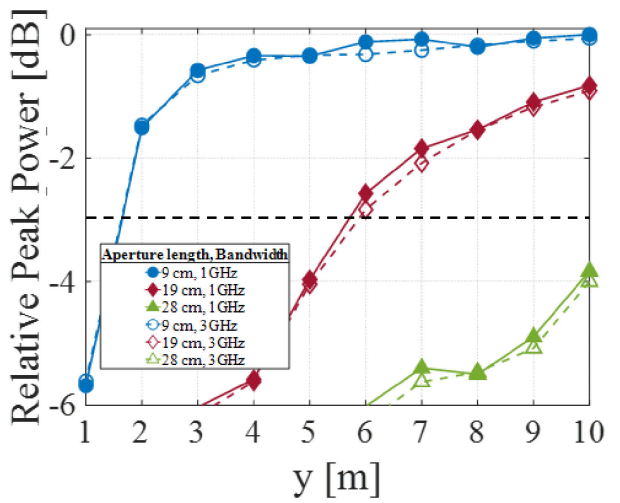

(a) Far-field mode vector

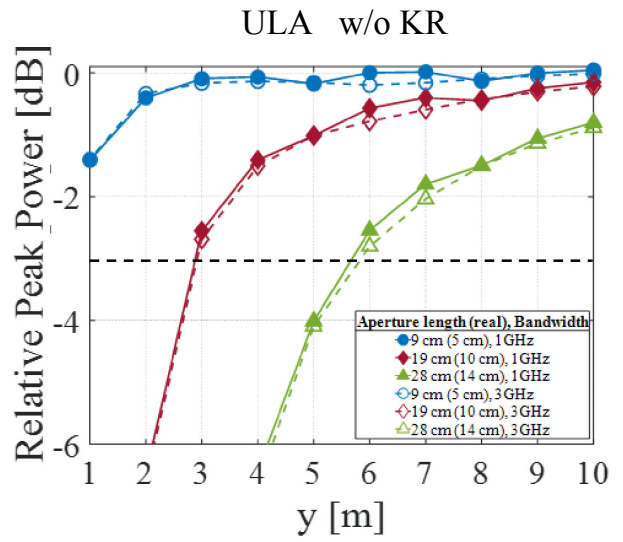

(c) Far-field mode vector LRLA w/ KR

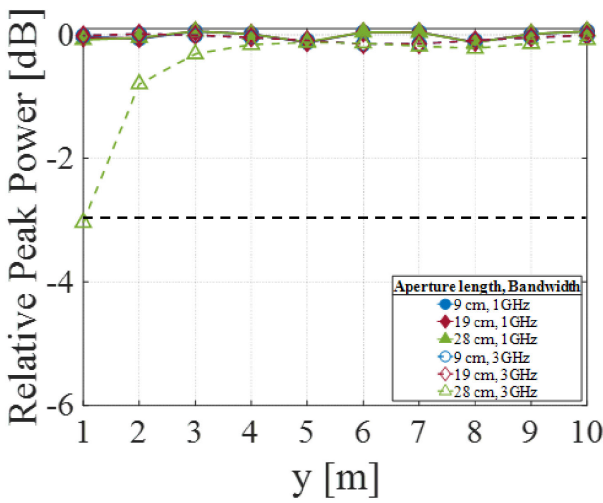

(b) Near-field mode vector ULA w/o KR.

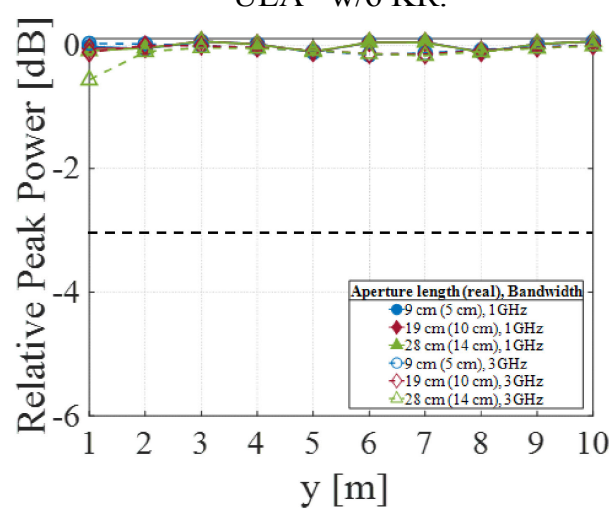

(d) Near-field mode vector LRLA w/KR.

Fig. 3. Evaluation of mode vector.

\section{Conclusion}

In this report, we have evaluate imaging quality for short-range MW-SAR. The results show that the imaging quality can be good by using the near-field mode vector without range migration, as in the case of the situation put the target in $3 \mathrm{~m}$ $(750 \lambda)$ with $28 \mathrm{~cm}(70 \lambda)$ aperture. It was also confirmed that a narrower frequency bandwidth provided better results. Furthermore, we showed that the imaging quality can be improved by the virtual array using the KR processing. This evaluation is applicable for 2D MIMO radar. It will be done in near future.

\section{Acknowledgments}

This work was partly supported by the JSPS KAKENHI Grant number 17H03261, Japan. 